\title{
Aspectos epidemiológicos e assistenciais de pacientes neurológicos em Unidade de Terapia Intensiva
}

\author{
Epidemiological and care aspects of neurological patients in \\ Intensive Care Unit
}

\section{Aspectos epidemiológicos y de cuidado de los pacientes neurológicos en una unidad de cuidados intensivos}

\author{
Naiara Kássia Macêdo da Silva Bezerra ${ }^{1}$, Laize Damasceno Argolo², \\ Marília Carolina dos Santos Lino ${ }^{3}$, Lilian Ramine Ramos de Souza \\ Matos $^{4}$, Márcia Bento Moreira ${ }^{5}$
}

\begin{abstract}
1.Fisioterapeuta e Docente, Mestre em Ciências pelo PPGCSB da UNIVASF, Unidade de Reabilitação/UTI, HU-UNIVASF, Petrolina-PE, Brasil. https://orcid.org/0000-0001-7271-8626

2.Discente do colegiado de Fisioterapia, Faculdade São Francisco de Juazeiro, Juazeiro-BA, Brasil. https://orcid.org/0000-0001-7040-6346

3.Discente do colegiado de Fisioterapia, Faculdade São Francisco de Juazeiro, Juazeiro-BA, Brasil. https://orcid.org/0000-0002-3992-429X

4.Fisioterapeuta e Docente, Mestre em Ciências, Unidade de Reabilitação, HU-UNIVASF, Petrolina-PE, Brasil. https://orcid.org/0000-0001-6588-4781

5. Médica Veterinária e Docente. Doutora em Cirurgia e Experimentação pela UNIFESP, Programa de Pós Graduação Ciências da Saúde e Biológicas, UNIVASF, Petrolina-PE, Brasil. https://orcid.org/0000-00024149-097X
\end{abstract}

\section{Resumo}

Introdução. Lesões neurológicas resultam em incapacidades graves, no início do diagnóstico podem apresentar estado geral grave e necessitar de cuidados intensivos. A frequência com que os pacientes neurológicos são internados e suas evoluções são pouco conhecidas, além de peculiaridades regionais, importante para prognóstico e medidas intervencionistas. Objetivo. Descrever o perfil de saúde de pacientes neurológicos internados em unidade de terapia intensiva (UTI), com destaque para os aspectos epidemiológicos e indicadores assistenciais de um hospital universitário, referência de atendimento da região do submédio Vale do São Francisco. Método. Estudo transversal com dados de prontuários de pacientes internados na UTI, no período de janeiro a outubro de 2018. As variáveis foram analisadas no programa SPSS. Resultados. Foram analisados 140 prontuários, predominando a população do sexo masculino e adultos-jovens. As principais causas de internação foram o traumatismo cranioencefálico, hematomas cerebrais e acidente cerebrovascular. O tempo médio de internação foi de 12 dias e o tempo de assistência ventilatória mecânica foi de 11 dias. Conclusão. O conhecimento desses dados é fundamental para aperfeiçoar o atendimento ao paciente neurológico na UTI. Espera-se enriquecer a comunidade cientifica com as informações do perfil traçado e orientar políticas de saúde e estratégias para melhorar o cuidado desses pacientes.

Unitermos. Acidente Cerebrovascular; Traumatismo Cranioencefálico; Lesões Cerebrais; Epidemiologia; UTI; Perfil de saúde

\footnotetext{
Abstract

Introduction. Neurological injuries result in severe disabilities, at the beginning of the diagnosis they may present a severe general condition and require intensive care. The frequency with which neurological patients are hospitalized and their evolution are little known, in addition to regional peculiarities, important for prognosis and interventionist measures. Objective. Describe the health profile of neurological patients hospitalized in an intensive care unit (ICU), with emphasis on the epidemiological aspects and care indicators of a university
} 
hospital, a reference in the region of the Vale do São Francisco. Method. Cross-sectional study with data from medical records of patients admitted to the ICU, from January to October 2018. The variables were analyzed in the SPSS program. Results. 140 medical records were analyzed, with a predominance of the male population and young adults. The main causes of hospitalization were traumatic brain injury, brain hematomas and stroke. The average hospital stay was 12 days and the duration of mechanical ventilatory assistance was 11 days. Conclusion. Knowledge of these data is essential to improve care for neurological patients in the ICU. It is hoped to enrich the scientific community with information from the outlined profile and to guide health policies and strategies to improve the care of these patients.

Keywords. stroke; Cranioencephalic trauma; Brain Injuries; Epidemiology; ICU; Health profile

\section{Resumen}

Introducción. Las lesiones neurológicas resultan en discapacidades severas, al comienzo del diagnóstico pueden presentar una condición general severa y requieren cuidados intensivos. La frecuencia con la que los pacientes neurológicos son hospitalizados y su evolución son poco conocidos, además de las peculiaridades regionales, importantes para el pronóstico y las medidas intervencionistas. Objetivo. Describa el perfil de salud de los pacientes neurológicos hospitalizados en una unidad de cuidados intensivos (UCI), con énfasis en los aspectos epidemiológicos y los indicadores de atención de un hospital universitario, una referencia en la región del submedio Vale do São Francisco. Método. Estudio transversal con datos de registros médicos de pacientes ingresados en la UCI, de enero a octubre de 2018. Las variables se analizaron en el programa SPSS. Resultados. Se analizaron 140 registros médicos, con predominio de la población masculina y adultos jóvenes. Las principales causas de hospitalización fueron lesión cerebral traumática, hematomas cerebrales y accidente cerebrovascular. La estancia hospitalaria promedio fue de 12 días y la duración de la asistencia ventilatoria mecánica fue de 11 días. Conclusión. El conocimiento de estos datos es esencial para mejorar la atención de los pacientes neurológicos en la UCI. Se espera enriquecer a la comunidad científica con información del perfil descrito y guiar las políticas y estrategias de salud para mejorar la atención de estos pacientes.

Palabras clave. Accidente cerebrovascular; Traumatismo craneoencefálico; Lesiones cerebrales; Epidemiología; UCI; Perfil de salud

Trabalho realizado no Hospital Universitário da Universidade do Vale do São Francisco, Petrolina-PE, Brasil.

\section{INTRODUÇÃO}

O paciente com uma condição neurológica crítica é todo aquele que sofre uma lesão neurológica grave e cursa muitas vezes com redução no nível de consciência e deficiências do movimento, coordenação e consequente debilidade funcional, e por muitas vezes se encontram em situações de risco para lesões neurológicas irreversíveis, necessitando ter 
à disposição uma equipe multiprofissional capacitada e especializada para seu tratamento ${ }^{1}$.

Diante desta necessidade surge o neurointensivismo, que vem crescendo como especialidade na Unidade de Terapia Intensiva (UTI) no Brasil e no mundo. A UTI proporciona cuidados imediatos após a lesão, na diligência de reverter o quadro com a monitorização em tempo integral e suporte ventilatório. Esse suporte de cuidado demanda estratégias em saúde que devem ser adequadas às diferentes realidades do país, o que repercute na melhora funcional do paciente, na diminuição do tempo de permanência hospitalar, na redução da mortalidade e na otimização de recursos².

Identificar as peculiaridades regionais associadas a este perfil de paciente com disfunção neurológica que necessitam do cuidado na UTI é importante para estabelecer o prognóstico e medidas intervencionistas específicas nessa população. Os dados coletados podem propiciar comparações com outras unidades congêneres, sejam elas regionais ou internacionais, ou até mesmo com a própria unidade para comparações futuras de modo que seja possível uma busca incessante da qualidade na atenção à saúde ${ }^{3}$.

Um crescente corpo de estudos demonstra que o internamento prolongado na UTI, frequentemente têm significativas alterações neuromusculares e complicações que prejudicam o desenvolvimento físico-funcional, implicando em maior tempo de ventilação mecânica (VM) ou 
suporte ventilatório mecânico, aumento do tempo de permanência na unidade, maiores custos assistenciais, recuperação funcional fraca e redução do retorno ao trabalho entre pacientes que recebem alta ${ }^{4-6}$.

Estima-se que milhões de pessoas vivam com sequelas neurológicas irreversíveis acarretando significativo impacto financeiro, diante da alta prevalência e taxas de incidência em constante elevação. Desta forma, o objetivo deste estudo é descrever o perfil de saúde de pacientes neurológicos internados em unidade de terapia intensiva, com destaque para os aspectos epidemiológicos e indicadores assistenciais.

\section{MÉTODO}

\section{Amostra}

Trata-se de um estudo observacional, transversal de abordagem exploratória, realizado no Hospital Universitário da Universidade Federal do Vale do São Francisco (HU/UNIVASF), em Petrolina, por meio de análise de prontuários de pacientes com doença neurológica internados na UTI geral, durante o período de 01 de janeiro a 31 de outubro de 2018. O local foi escolhido, pois o HU-UNIVASF é referência em atendimento neurológico na região do vale do Submédio São Francisco.

Para a execução da presente pesquisa, foram respeitados os princípios éticos adotados na Resolução No 466/12 do Conselho Nacional de Saúde que regulamenta a pesquisa envolvendo seres humanos. $O$ projeto foi 
submetido ao Comitê Institucional de Ética em Pesquisa (CEP) da Universidade Federal do Vale do São Francisco (UNIVASF) e aprovado, sob o parecer no 2.759.229.

\section{Procedimento}

Foram coletadas variáveis epidemiológicas: gênero biológico e idade e dentre as principais variáveis clínicas obteve-se o diagnóstico neurológico primário e os indicadores de evolução clínica da assistência, como: tempo de internamento na UTI, uso de ventilação mecânica (VM), alta da UTI ou óbito (taxa de mortalidade).

Obedeceram aos critérios de inclusão na pesquisa: prontuários de pacientes admitidos na UTI do HU/UNIVASF, de ambos os gêneros, com tempo de permanência na UTI $>24 \mathrm{~h}$ e idade acima de dezoito anos, cujo motivo de internação foi por diagnóstico neurológico primário. Para não haver perda amostral e reduzir o risco de viés de publicação, foram incluídos dados em que houve perda de seguimento das informações do objeto de estudo, sendo aproveitado os dados relevantes, ao invés de perda total do prontuário do paciente.

\section{Análise Estatística}

Todos os dados obtidos foram organizados em planilha no Microsoft Office Excel 2016, os quais foram analisados por meio de estatística descritiva, através das medidas de tendência central e frequência absoluta e relativa, com a utilização do programa Statistical Package for the Social 
Science (SPSS) e os resultados expressos em formato de tabelas e gráficos.

\section{RESULTADOS}

O total de prontuários analisados foi obtido através do registro de informações de pacientes em um caderno ata, realizado a cada admissão pela equipe de enfermagem da UTI. A distribuição do total de admissões nessa unidade está expressa no fluxograma apresentado na Figura 1.

Figura 1. Fluxograma de seleção dos dados de prontuários para análise.

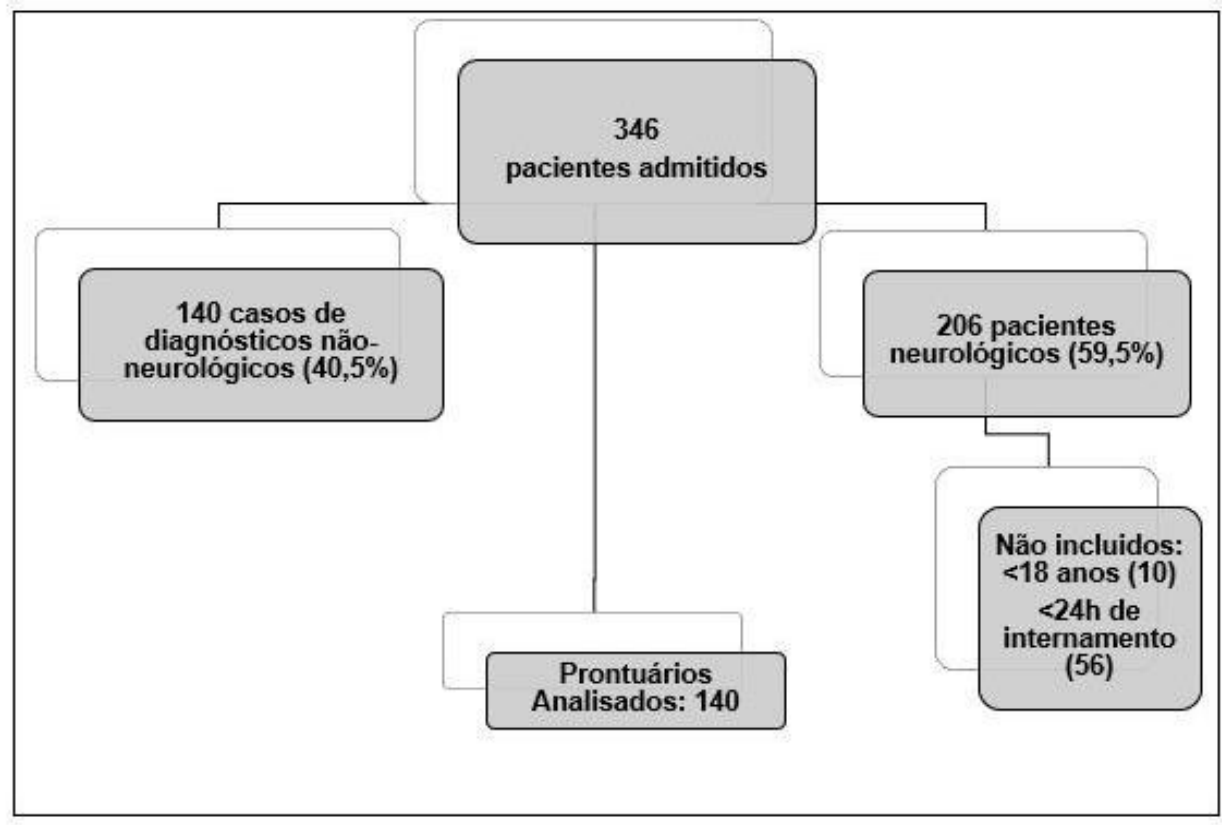

Dentre os prontuários, 59,5\% $(\mathrm{n}=206)$ dos pacientes admitidos foram devido a condição neurológica e após 
aplicação dos critérios de não-inclusão, participaram da composição do perfil dados de um total de 140 pacientes.

Referente ao gênero biológico, 66\% $(n=93)$ eram do gênero masculino e 34\% $(n=47)$ do gênero feminino.

A média de idade dos indivíduos foi de 44 anos, variando de 18 a 89 anos, com a faixa etária mais prevalente entre 29 a 40 anos $(n=35)$, mas vale ressaltar que mais de 80 pacientes possuíam menos de 50 anos (Gráfico 1).

Gráfico 1. Distribuição das internações por intervalos de idades.

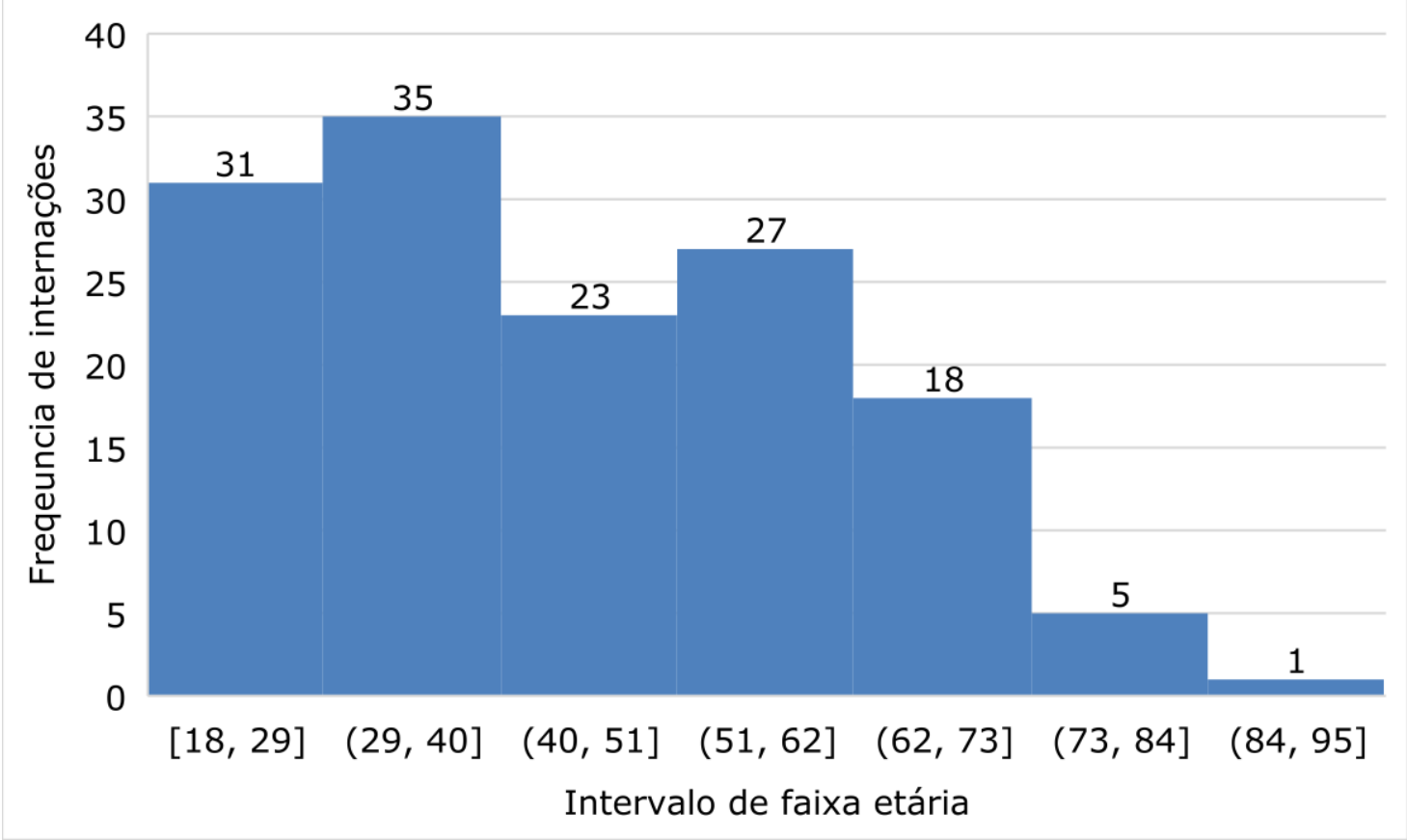

Dentre os principais diagnósticos neurológicos encontram-se os hematomas cerebrais, tumores cerebrais, mas, a causa primária que levou à internação na UTI foi o TCE grave, representando $40 \%$ dos casos. O acidente 
vascular cerebral (AVC) aparece com cerca de $12 \%$ dos casos (Tabela 1 ).

Tabela 1. Descrição dos principais diagnósticos neurológicos $(n=140)$.

\begin{tabular}{lcc}
\hline \multicolumn{1}{c}{ Diagnóstico } & Quantidade & \% \\
\hline Traumatismo cranioencefálico & 56 & 40,00 \\
Hematomas cerebrais & 24 & 17,14 \\
Acidente vascular encefálico & 17 & 12,14 \\
Tumor cerebral & 15 & 10,72 \\
Hemorragia subaracnóide & 7 & 5,00 \\
Craniectomia descompressiva & 6 & 4,29 \\
Clipagem de aneurisma & 5 & 3,57 \\
Hidrocefalia & 4 & 2,86 \\
Meningite & 3 & 2,14 \\
Trauma raquimedular & 2 & 1,43 \\
Trombose venosa cerebral & 1 & 0,71 \\
\hline
\end{tabular}

Referente aos indicadores de evolução clínica da assistência constatou-se que os tempos médios das principais variáveis foram: 12,4 dias de permanência na UTI; 11 dias de ventilação mecânica (VM) e os pacientes receberam alta em média 2,3 dias após o desmame da VM (Gráfico 2).

No desfecho alta ou óbito, um total de 103 (74\%) indivíduos obtiveram alta da Unidade de Terapia Intensiva e 37 (26\%) foram a óbito, apresentando uma taxa de mortalidade maior no sexo masculino, com $65 \%$ do total de óbitos (Tabela 2). 
Gráfico 2. Distribuição dos indicadores assistenciais na UTI, em média de dias.

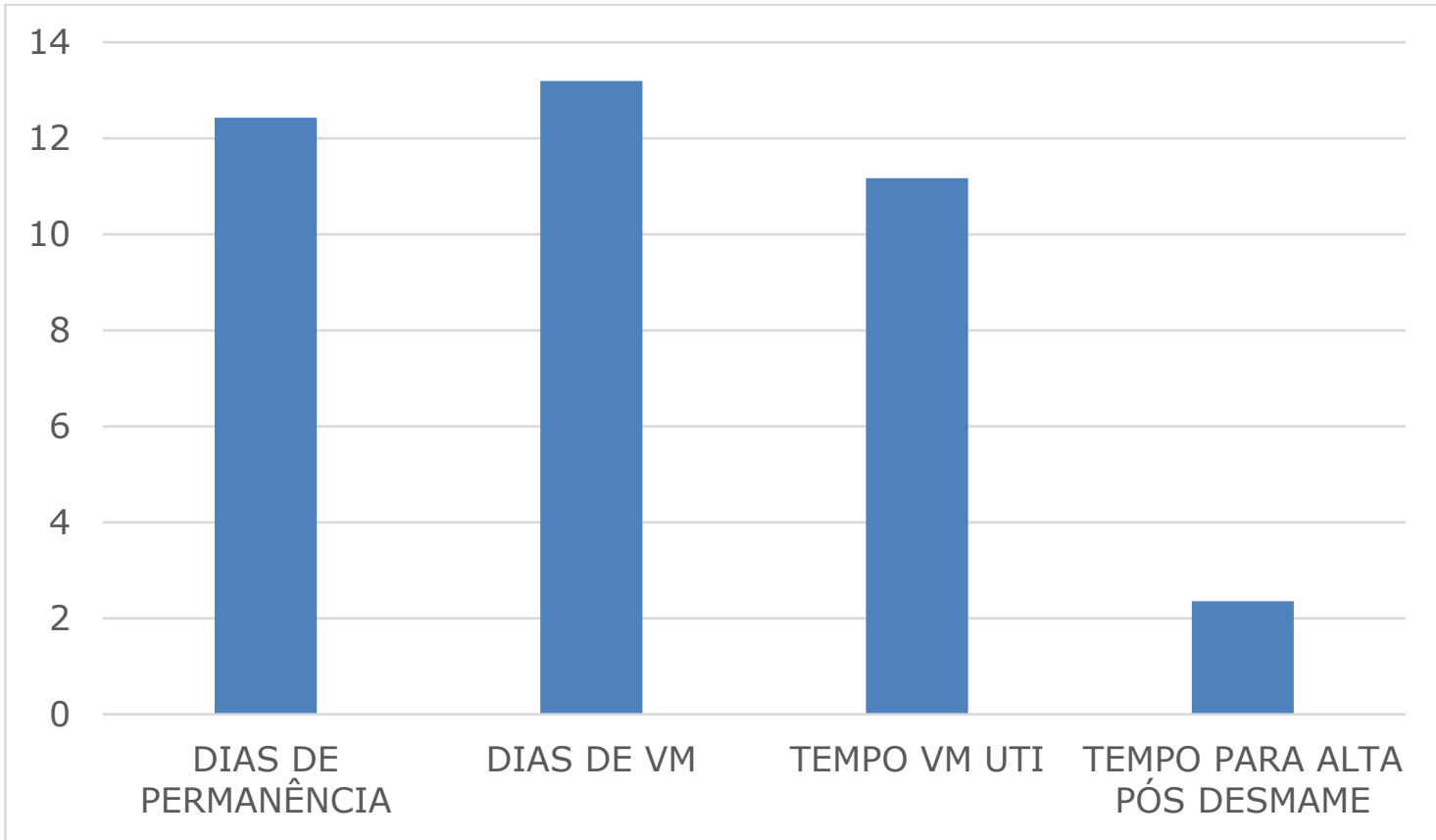

VM: ventilação mecânica; UTI: unidade de terapia intensiva.

Tabela 2. Caracterização geral do perfil epidemiológico.

\begin{tabular}{lll}
\hline Variáveis & Total (n) & \% \\
\hline $\begin{array}{l}\text { Gênero biológico } \\
\text { Feminino } \\
\text { Masculino }\end{array}$ & 47 & 34 \\
Total & 93 & 66 \\
\hline Desfecho & $\mathbf{1 4 0}$ & \\
Alta & Óbito & 74 \\
Total & 103 & 26 \\
\hline Mortalidade por gênero & 37 & \\
$\quad$ Feminino & $\mathbf{1 4 0}$ & 35 \\
$\quad$ Masculino & 13 & 65 \\
Total & 24 & \\
\hline
\end{tabular}

\section{DISCUSSÃO}

Os resultados aqui descritos encontraram uma alta prevalência de admissões de pessoas com disfunções 
neurológicas $(59,5 \%)$, contrastando com uma pesquisa com o mesmo objetivo em um hospital de atenção terciária na cidade do Rio de Janeiro, na qual analisaram 600 prontuários e observaram um percentual de $19 \%$ de diagnósticos neurológicos 5 .

Esse discordância pode ter interferência de aspectos culturais, hábitos de vida e diferenças geográficas, além do perfil institucional, pois neste trabalho, o HU-UNIVASF é referência regional e interestadual em atendimento a politraumatizados para cerca de 53 municípios, sendo porta de entrada para casos de urgência e emergência e ofertando atendimentos em neurocirurgia, neuroclínica, ortopedia, cirurgia geral e vascular, dentre outros.

Em relação ao gênero biológico, um estudo que identificou as características epidemiológicas e causas de óbitos em pacientes internados em terapia intensiva de um hospital que atende exclusivamente pelo Sistema Único de Saúde (SUS), localizado na cidade de Florianópolis - Santa Catarina, verificou que de 695 prontuários analisados, $61,6 \%$ dos pacientes eram do gênero masculino ${ }^{6}$, corroborando com os dados encontrados no presente trabalho.

Segundo o Ministério da Saúde, os homens adoecem mais do que as mulheres por não procurarem os serviços de saúde por medo de descobrir as doenças, além de estarem mais expostos aos acidentes de trabalho e de trânsito por utilizar álcool e drogas com maior frequência, além de estarem envolvidos na maioria das situações de violência?. 
Quanto à idade dos pacientes, observou-se um acometimento maior de adultos jovens, o que corrobora com dados publicados em 2014, onde foram identificados 361.577 óbitos na faixa etária de 20 a 59 anos no Brasil e se correlacionado com o gênero biológico, 68\% dessas mortes foram em homens ${ }^{8}$.

Em um panorama mundial, dentre as principais condições neurológicas que geram internamento merecem destaque $o$ acidente vascular cerebral (AVC) que permanece sendo a primeira causa de morte e incapacidade em idosos no Brasil ${ }^{9}$ e o traumatismo cranioencefálico (TCE) que é considerado uma importante causa de morte e incapacidade em todo mundo, acometendo principalmente adultos jovens ${ }^{10}$. Essa informação ratifica os resultados do diagnóstico clínico primário, em que o TCE grave representou $40 \%$ dos casos e o AVC apareceu com $12 \%$.

Os hematomas cerebrais que aparecem em segunda causa de internação nesta pesquisa podem ainda aumentar o percentual de internações por TCE grave, uma vez que os hematomas cerebrais podem acontecer em decorrência de traumas diretos, tendo uma associação com o crescente número de internações nas UTI por TCE.

Ao identificar a média de 12,4 dias de internamento e 11 dias de VM na UTI do HU/UNIVASF, pode-se observar o contraste com um estudo multicêntrico realizado desde 2010 pela Associação de Medicina Intensiva Brasileira (AMIB), a qual objetivou confeccionar o perfil das UTI brasileiras e verificou que em 2018 a duração média das internações nas 
UTI's brasileiras foi de 5,58 dias, e nos hospitais públicos chegou a 7,46 dias ${ }^{11}$. Presume-se que a divergência de resultados encontrados possa ter relação com o perfil de pacientes internados, o que interfere diretamente no tempo desprendido de suporte ventilatório e consequentemente nos dias de internamento.

Faz-se necessário também averiguar sobre as tecnologias utilizadas, a qualificação da equipe, o preparo para receber pacientes neurológicos, bem como os protocolos de desmame ventilatório utilizados, pois um dado notório foi a média de dias de internamento e de uso de VM, ou seja, mais de $95 \%$ do tempo em uso, alta da UTI após cerca de 2 dias sem o uso desse recurso. Esses dados podem inferir que o uso do suporte ventilatório pode prolongar os dias de permanência dos pacientes na UTI, o que majora efeitos adversos decorrentes do imobilismo, bem como os custos em assistência nos serviços.

No tocante ao desfecho alta ou óbito, merece destaque que $74 \%$ dos pacientes tiveram alta da unidade e a taxa de mortalidade foi de $26 \%$. Sobre isto, uma pesquisa observouse que a gravidade da doença apresentada pelo paciente influencia diretamente tanto no tempo de permanência na UTI do doente, quanto em sua evolução para alta ou óbito ${ }^{12,13}$, o que provavelmente está relacionado também aos achados do presente estudo, pois a lesão neurológica apresenta risco iminente de vida ao ser humano.

Estudiosos afirmam que $\mathrm{o}$ cuidado de pacientes neurológicos graves ainda encontra muitas limitações, como 
por exemplo: ambientes e cuidados intensivos limitados, falta de investimento em políticas públicas que tem uma relação direta com a escassez de pesquisas e dados epidemiológicos ${ }^{14}$.

Com isso, observa-se a necessidade de uma capacitação efetiva da equipe multiprofissional envolvida no cuidado desses pacientes, para que sejam elaborados fluxogramas e protocolos de atendimento, visando uma melhor oferta de sobrevida ao paciente e otimizando a gestão de leitos e recursos hospitalares, além da repercussão direta nos desfechos de evolução clínica do doente.

\section{CONCLUSÃO}

O traçado do perfil de saúde de pacientes neurológicos em Unidade de Terapia Intensiva permite delimitar a magnitude das condições de saúde e suas repercussões, e dessa forma possibilitar o direcionamento de políticas de saúde, sobretudo local e regional, visando o fortalecimento de estratégias voltadas ao cuidado destes, trazendo como consequência a redução de gastos públicos.

\section{REFERÊNCIAS}

1.Pereira JA, Guedes N. Reabilitação precoce no doente com lesão cerebral aguda. Rev Port Med Int 2011;18:81-7.

https://enfermeiros-intensivistas.webnode.pt/_files/200000338-

64648655ea/CLIQUE\%20AQUI\%20RPMI_2011_V_18_03.pdf

2. Diccini S. Desafio das unidades de cuidados neurointensivos. Acta Paul Enferm 2012;25:8-9. http://dx.doi.org/10.1590/S010321002012000800001

3. Lanetzki CS, Oliveira CAC, Bass LM, Abramovici S, Troster EJ. O perfil epidemiológico do Centro de Terapia Intensiva Pediátrico do Hospital Israelita Albert Einstein. Einstein 2012;10:16-21. http://dx.doi.org/10.1590/S1679-45082012000100005 
4.França EET, Ferrari F, Fernandes $P$, Cavalcanti R, Duarte $A$, Martinez $\mathrm{BP}$, et al. Fisioterapia em pacientes críticos adultos: recomendações do Departamento de Fisioterapia da Associação de Medicina Intensiva Brasileira. Rev Bras Ter Intensiva 2012;24:6-22. http://dx.doi.org/10.1590/S0103-507X2012000100003

5.Gosselink R, Bott J, Johnson M, Dean E, Nava S, Norrenberg M, et al. Physiotherapy for adult patients with critical illness: recommendations of the European Respiratory Society and European Society of Intensive Care Medicine Task Force on Physiotherapy for Critically Ill Patients. Intensive Care Med 2008;34:1188-99. http://dx.doi.org/10.1007/s00134-008-1026-7

6. Hodgson C, Bellomo R, Berney S, Bailey M, Buhr H, Denehy L, et al. Early mobilization and recovery in mechanically ventilated patients in the ICU: a bi-national, multi-centre, prospective cohort study. Crit Care 2015;19:81. http://dx.doi.org/10.1186/s13054-015-0765-4

7.Albuquerque JM, Silva RFA, Souza RFF. Perfil epidemiológico e seguimento após alta de pacientes internados em unidade de terapia intensiva. Cogitare Enferm 2017;22:1-9. http://dx.doi.org/10.5380/ce.v22i3.50

8.Rodriguez AH, Bub MBC, Perão OF, Zandonadi G, Rodriguez M JH. Características epidemiológicas e causas de óbitos em pacientes internados em terapia intensiva. Rev Bras Enferm 2016;69:229-34. http://dx.doi.org/10.1590/0034-7167.2016690204i

9.Política Nacional de Atenção à saúde do Homem (Princípios e Diretrizes) (endereço na Internet). Brasil: Ministério da Saúde. (atualizado em: 2008, assessado em 03/2020). Disponivel em: https://bvsms.saude.gov.br/bvs/publicacoes/politica_nacional_atenca o_homem.pdf

10.Araújo JP, Darcis JVV, Tomas ACV, Mello WA. Tendência da Mortalidade por Acidente Vascular Cerebral no Município de Maringá, Paraná entre os Anos de 2005 a 2015. Int J Cardiovasc Sci 2018;31:5662. http://dx.doi.org/10.5935/2359-4802.20170097

11.Magalhães ALG, Souza LC, Faleiro RM, Teixeira AL, Miranda AS. Epidemiologia do traumatismo cranioencefálico no Brasil. Rev Bras Neurol 2017;53:15-22.

https://revistas.ufrj.br/index.php/rbn/article/view/12305

12.UTIs brasileiras: Registro Nacional de Terapia Intensiva (endereço na Internet). AMIB-Associação de Medicina Intensiva Brasileira. (assessado 09/2019). Disponível em: http://www.utisbrasileiras.com.br/o-projeto/

13. Melo EM, Santos AMM, Silveira FMM, Sombra RLS, Alves RL, Lima VF. Clinical and demographic characteristics of patients on mechanical ventilation in the intensive care unit. Rev Enferm UFPI 2015;4:36-41. http://dx.doi.org/10.26694/reufpi.v4i3.3599

14.Shrestha GS, Goffi A, Aryal D. Delivering neurocritical care in resource-challenged environments. Curr Opin Crit Care 2016;22:1005. http://dx.doi.org/10.1097/MCC.0000000000000285 